See discussions, stats, and author profiles for this publication at: https://www.researchgate.net/publication/260538433

\title{
Ethernet-OCDMA system for multi-user visible light communications
}

Article in Electronics Letters · February 2012

DOI: 10.1049/el.2011.4035

\section{CITATIONS}

50

6 authors, including:

(6) Marcos F. Guerra-Medina 9 PUBLICATIONS 106 CITATIONS

SEE PROFILE

J. Rabadan

Universidad de Las Palmas de Gran Canaria

58 PUBLICATIONS 703 CITATIONS

SEE PROFILE

Some of the authors of this publication are also working on these related projects:

Optimal Signal Processing in Visible Light Communications View project

Project Spread spectrum for wireless optical systems View project
Universidad de Las Palmas de Gran Canaria

29 PUBLICATIONS 281 CITATIONS

SEE PROFILE 


\section{Ethernet-OCDMA system for multi-user visible light communications}

M.F. Guerra-Medina, O. González, B. Rojas-Guillama, J.A. Martín-González, F. Delgado and J. Rabadán

\begin{abstract}
An optical code-division multiple access system (OCDMA) for multiuser visible light communications is presented, which interfaces with the Ethernet network. The OCDMA scheme is based on the optical codes known as random optical codes, which do not present an optimal performance but are characterised by their ease of implementation. To combat the relative poor correlation properties of this kind of code, a synchronism mechanism has been proposed and evaluated. The results obtained with the final developed prototype demonstrate the suitability of the proposed scheme for multi-user communications in a visible light environment
\end{abstract}

Introduction: In recent years, visible LED lamps have been proposed for the simultaneous transmission of information, which is known as visible light communications (VLC) [1]. These new VLC systems share the same advantages as their infrared counterparts [2], but they are also eye-safe (visible light is not prejudicial to the human eye), which enables the use of higher transmission powers. However, the main drawback is the limited transmission bandwidth of current LED devices [3]

Until now, most of the presented works are concerned with broadcasting information $[1,3]$. In this Letter, we present an OCDMA system for visible light communications that allows for simultaneous transmission from several sources by using random optical codes (ROCs) as coding sequences [4]. ROCs have been devised for applications where a large number of users are sharing the channel, and they are characterised by their ease of generation but little performance degradation [4] with respect to other more optimal codes, such as optical orthogonal codes (OOC) [5]. Moreover, ROCs can be easily adapted to the bit error rate (BER) requirements when the number of users varies [6], which makes them very useful for the implementation of this kind of changeable wireless network.

System description: In OCDMA, a code sequence is assigned to each user, being the bit period $T$ divided in $L$ time slots or chips, each of duration $T_{c}\left(T=L T_{c}\right)$. The parameter $L$ is the length of the code and, during the bit period, only $w$ pulses (chips) are actually transmitted, with $w<<$ $L$. The parameter $w$ is called the weight of the code and, for ROCs, the position of the $w$ pulses or chips is randomly placed in $L$. Moreover, the code sequence is only transmitted when the data bit is a ' 1 '. On the other hand, no signal is transmitted when the data bit is a ' 0 '. The bit error rate $\left(P_{e}\right)$ for ROCs converges to [4]

$$
P_{e}=\frac{1}{2^{s}} \sum_{i=0}^{s-1}\left(\begin{array}{c}
s-1 \\
i
\end{array}\right)\left[1-\left(1-\frac{w}{L}\right)^{i}\right]^{w}
$$

where $s$ is the number of simultaneous users sharing the channel, and $L$, $w$ are the parameters of the codes. A synchronism mechanism has been devised for this kind of code which consists of sending a synchronisation preamble of $m$ bits previously to transmit the normal data. The proposed method can be described as follows: the emitter sends a normal data bit ' 1 ' ( first synchronisation bit) and, afterwards, each new $l$ th synchronisation bit (for $1 \leq l \leq m-1$, the index $l=0$ refers to the first non-rotated synchronisation bit) is circularly shifted $i \times l$ chips with respect to the previous synchronisation bit, for the $i$ th user (see Fig. 1). We have to take into account that the corresponding receiver is looking for $w$ pulses with a relative delay between them according to its code sequence. Therefore, if several users are transmitting simultaneously, they can insert pulses into the channel with the same relative delay as the intended code. This is known as a 'false alarm' or 'synchronisation error'. However, by transmitting $m$ instead of only one code sequence, the interference pattern due to the $s-1$ remaining users is less probable, because it would have to occur in all the $m$ synchronisation bits Furthermore, by circularly shifting the codes, if an interference pattern produces a 'false alarm' in one of the synchronisation bits, the act of reordering the chips newly, the shift being different for each user (for example, when all the users are trying to synchronise simultaneously, the worst case of interference), prevents, with a high probability, the possibility of a new 'false alarm' in the remaining $m-1$ synchronisation bits, especially when $m$ is large enough. It has been shown by simulation that for $m \geq 3$, the probability of a 'synchronisation error' is inferior to the BER of the system during normal transmission, as the experimental results have stated (see following Section). Therefore, the proposed synchronisation mechanism is highly reliable, in spite of the poor correlation properties of ROCs.

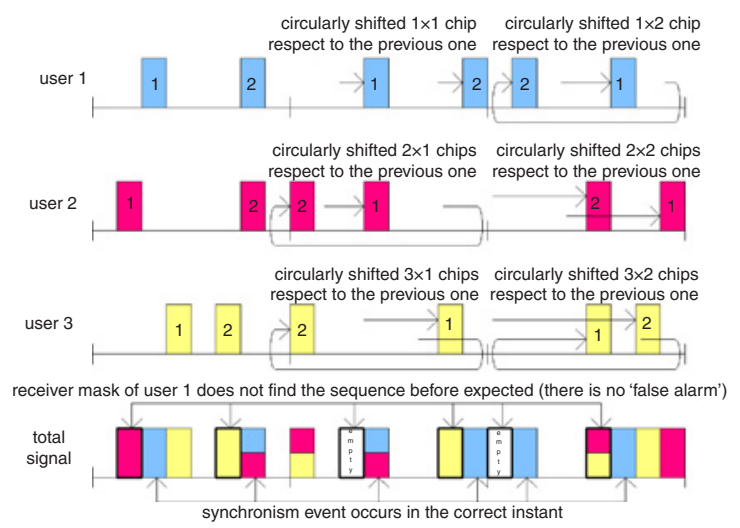

Fig. 1 Illustration of proposed circular shift mechanism and its efficiency to avoid 'false alarms' (example for $s=3$ users, $m=3$ synchronisation bits and code parameters $L=8$ and $w=2$ )

Results: An experimental prototype has been developed (Fig. 2), where the optical link has been implemented by using visible LED transmitters and pin photodiodes (Hamamatsu S7510) in combination with a transimpedance stage. The prototype allows for the optical transmission of messages constituted by a synchronisation preamble, a data field of variable length and a checksum field for bit error detection. It has also been provided with a fast Ethernet module, which allows for the reception and transmission of UDP/IP packets through the Ethernet LAN network. Thus, the received UDP packets can be OCDMA encoded and optically transmitted, whereas the received OCDMA messages can be newly encapsulated in UDP packets to be transmitted through the LAN. All the digital logic (OCDMA encoders and decoders, synchronisers, UDP/IP/Ethernet modules, etc.) have been implemented inside Xilinx ${ }^{\circledR}$ Virtex-5 FPGAs. Fig. 3 shows a snapshot of some signals of the prototype (obtained with a digital oscilloscope) during a communication. We can see how the corresponding receiver detects the synchronisation header of a message, generating a synchronism event, and the data are demodulated correctly.

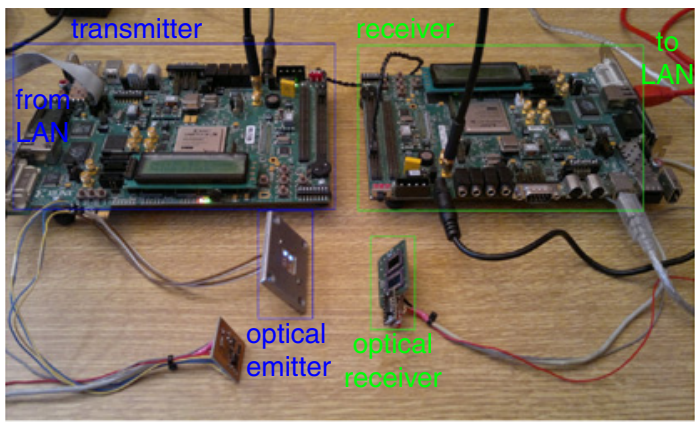

Fig. 2 Experimental prototype (optical emitter and receiver have been brought closer to take this photo, but more than $1 \mathrm{~m}$ distances have been demonstrated, obtaining perfect performance of system using only two low-power visible LEDs; commercial LED lamps contain tens of these devices)

Several tests have been carried out over the developed prototype to evaluate its performance. The parameters of the codes were length $L=100$ and weight $w=10$, with $s=8$ simultaneous users (channels). The chip rate was $R_{\text {chip }}=2$ Mchips/s, leading to a bit rate of $R=20$ kbit/s per channel (total throughput: $160 \mathrm{kbit} / \mathrm{s}$ ). During the trials (where each channel was continuously transmitting data at random instants), the observed BER was close to $2 \times 10^{-5}$, as expected according to (1). Moreover, for $m=3$ and 4 synchronisation bits, the synchronism error rate (SER) was below the BER as expected according to simulation studies, obtaining $5 \times 10^{-6}$ and no synchronisation errors, respectively (for $m=4$, the simulations indicated SER values 
of about $10^{-8}$ and only $10^{7}$ messages were sent during each experimental trial). Finally, the prototype was evaluated for the multi-user transmission of real-time audio data (eight channels), newly observing a high-quality reception practically error-free (BER of $\sim 10^{-5}$ was computed by means of the checksum information, although these errors were imperceptible to the human ear).

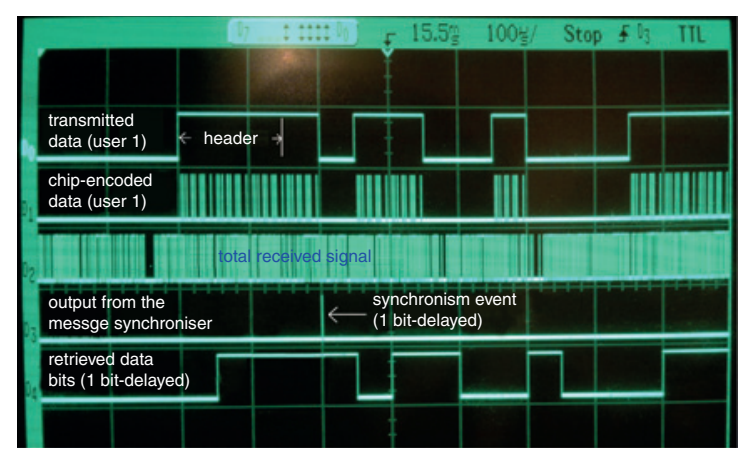

Fig. 3 Most significant signals in prototype during communication: transmitted and chip-encoded data bits for desirable user, total received signal (combination of those from all transmitting users), synchronism events and retrieved data bits at receiver

Conclusion: An OCDMA system has been experimentally developed and evaluated for visible light communications, which is provided with an Ethernet module for transmitting/receiving UDP packets throughout the wired LAN where it is connected. The prototype allows for multi-user communication at medium data rates by using the easily implementable ROCs and it uses a novel very robust synchronisation mechanism which enables a highly reliable optical transmission.

Acknowledgements: This work has been funded in part by the Canary Government (SolSubC20081000306) and the Spanish Research Administration (TEC2009-14059-C03-02/03).
(C) The Institution of Engineering and Technology 2012

23 December 2011

doi: $10.1049 / \mathrm{el} .2011 .4035$

One or more of the Figures in this Letter are available in colour online.

M.F. Guerra-Medina, O. González and B. Rojas-Guillama (Department of Fundamental and Experimental Physics, Electronics and Systems, University of La Laguna, Av. Francisco Sánchez S/N, La Laguna 38203, Spain)

E-mail: oghdez@ull.es

J.A. Martin-González (Department of Mathematics, University of Burgos, currently on secondment to CENIEH, Pza. Misael Bañuelos S/N, Burgos 09001, Spain)

F. Delgado and J. Rabadán (IDeTIC, University of Las Palmas de Gran Canaria, Campus de Tafira S/N, 35017, Las Palmas, Spain)

\section{References}

1 Elgala, H., Mesleh, R., and Haas, H.: 'Indoor optical wireless communication: potential and state-of-the-art', IEEE Commun. Mag., 2011, 49, (9), pp. 56-62

2 Kahn, J.M., and Barry, J.R.: 'Wireless infrared communications', Proc. IEEE, 1997, 85, (2), pp. 265-298

3 Grubor, J., Randel, S., Langer, K.-D., and Walewski, J.W.: 'Broadband information broadcasting using LED-based interior lighting', $J$. Lightwave Technol., 2008, 26, (24), pp. 3883-3892

4 Martín-González, J.A., Poves, E., and López-Hernández, F.J.: 'Random optical codes used in optical networks', IET Commun., 2009, 3, (8), pp. $1392-1401$

5 Salehi, J.A.: 'Emerging OCDMA communication systems and data networks', J. Opt. Netw., 2007, 6, (9), pp. 1138-1178

6 González, O., Martín-González, J.A., Poves, E., López-Hernández, F.J., and Pérez-Jiménez, R.: 'Adaptive code-division multiple-access system for communications over indoor wireless optical channels based on random optical codes', IET Optoelectron., 2009, 3, (4), pp. 187-196 